\title{
The Approximate Solution of Fractional Fredholm Integrodifferential Equations by Variational Iteration and Homotopy Perturbation Methods
}

\author{
Abdelouahab Kadem ${ }^{1}$ and Adem Kilicman ${ }^{2}$ \\ ${ }^{1}$ L.M.F.N Mathematics Department, University of Setif, Algeria \\ ${ }^{2}$ Department of Mathematics, Universiti Putra Malaysia, \\ 43400 Serdang, Selangor, Malaysia \\ Correspondence should be addressed to Adem Kilicman, akilicman@putra.upm.edu.my \\ Received 2 February 2012; Revised 20 February 2012; Accepted 23 March 2012 \\ Academic Editor: Bashir Ahmad
}

Copyright (C) 2012 A. Kadem and A. Kilicman. This is an open access article distributed under the Creative Commons Attribution License, which permits unrestricted use, distribution, and reproduction in any medium, provided the original work is properly cited.

Variational iteration method and homotopy perturbation method are used to solve the fractional Fredholm integrodifferential equations with constant coefficients. The obtained results indicate that the method is efficient and also accurate.

\section{Introduction}

The topic of fractional calculus has attracted many scientists because of its several applications in many areas, such as physics, chemistry, and engineering. For a detail survey with collections of applications in various fields, see, for example, [1-3].

Further, the fractional derivatives technique has been employed for solving linear fractional differential equations including the fractional integrodifferential equations; in this way, much of the efforts is devoted to searching for methods that generate accurate results, see $[4,5]$. In this work, we present two different methods, namely, homotopy perturbation method and variational iteration method [6], for solving a fractional Fredholm integrodifferential equations with constant coefficients. There is a vast literature, and we only mention the works of Liao which treat a homotopy method in $[7,8]$.

For the nonlinear equations with derivatives of integer order, many methods are used to derive approximation solution [9-14]. However, for the fractional differential equation, 
there are some limited approaches, such as Laplace transform method [3], the Fourier transform method [15], the iteration method [16], and the operational calculus method [17].

Recently, there has been considerable researches in fractional differential equations due to their numerous applications in the area of physics and engineering [18], such as phenomena in electromagnetic theory, acoustics, electrochemistry, and material science [3, $16,18,19]$. Similarly, there is also growing interest in the integrodifferential equations which are combination of differential and Fredholm-Volterra equations. In this work, we study these kind of equations that have the fractional order usually difficult to solve analytically, thus a numerical method is required, for example, the successive approximations, Adomian decomposition, Chebyshev and Taylor collocation, Haar Wavelet, Tau and Walsh series methods.

This note is devoted to the application of variational iteration method (VIM) and homotopy perturbation method (HPM) for solving fractional Fredholm integrodifferential equations with constant coefficients:

$$
\sum_{k=0}^{\infty} P_{k} D_{*}^{\alpha} y(t)=g(x)+\lambda \int_{0}^{a} H(x, t) y(t) d t, \quad a \leq x, t \leq b,
$$

under the initial-boundary conditions

$$
\begin{aligned}
& D_{*}^{\alpha} y(a)=y(0), \\
& D_{*}^{\alpha} y(0)=y^{\prime}(a),
\end{aligned}
$$

where $a$ is constant and $1<\alpha<2$ and $D_{*}^{\alpha}$ is the fractional derivative operator given in the Caputo sense. For the physical understanding of the fractional integrodifferential equations, see [20]. Further, we also note that fractional integrodifferential equations were associated with a certain class of phase angles and suggested a new way for understanding of Riemann's conjecture, see [21].

Outline of this paper is as follows. Section 2 contains preliminaries on fractional calculus. Section 3 is a short review of the homotopy method and Section 4 variational iteration method. Sections 5 and 6 are devoted to VIM and HPM analysis, respectively. Concluding remarks with suggestions for future work are listed in Section 7.

\section{Description of the Fractional Calculus}

In the following, we give the necessary notations and basic definitions and properties of fractional calculus theory; for more details, see [3, 13, 16, 22].

Definition 2.1. A real function $f(x), x>0$, is said to be in the space $C_{\alpha}, \alpha \in R$ if there exists a real number $(p>\alpha)$, such that $f(x)=x^{p} f_{1}(x)$, where $f_{1}(x)=C([0, \infty))$. Clearly, $C_{\alpha} \subset C_{\beta}$, if $\beta \leq \alpha$.

Definition 2.2. A function $f(x), x>0$, is said to be in space $C_{\alpha}^{m}, m \in N$, if $f^{(m)} \in C_{\alpha}$. 
Definition 2.3. The Riemann-Liouville fractional integral of order $\mu \geq 0$ for a function $f \in$ $C_{\alpha},(\alpha \geq 1)$ is defined as

$$
I^{\mu} f(t)=\frac{1}{\Gamma(\mu)} \int_{0}^{t}(t-\tau)^{\mu-1} f(\tau) d \tau, \quad \mu>0, t>0,
$$

in particular $I^{0} f(t)=f(t)$.

Definition 2.4. The Caputo fractional derivative of $f \in C_{-1}^{m}, m \in N$, is defined as

$$
D_{c}^{\mu} f(t)= \begin{cases}{\left[I^{m-\mu} f^{(m)}(t)\right],} & m-1<\mu \leq m, m \in N \\ \frac{d^{m}}{d t^{m}} f(t), & \mu=m .\end{cases}
$$

Note that

(i) $I^{\mu} t^{\gamma}=(\Gamma(\gamma+1) / \Gamma(\gamma+\mu+1)) t^{\gamma+\mu}, \mu>0, \gamma>-1, t>0$,

(ii) $I^{\mu}{ }^{C} D_{0^{+}}^{\mu} f(t)=f(t)-\sum_{k=0}^{m-1} f^{(k)}\left(0_{+}\right)\left(t^{k} / k !\right), m-1<\mu \leq m, m \in N$,

(iii) ${ }^{C} D_{0^{+}}^{\mu} f(t)=D^{\mu}\left(f(t)-\sum_{k=0}^{m-1} f^{(k)}\left(0_{+}\right)\left(t^{k} / k !\right)\right), m-1<\mu \leq m, m \in N$,

(iv)

$$
D^{\beta} I^{\alpha} f(t)= \begin{cases}I^{\alpha-\beta} f(t), & \text { if } \alpha>\beta, \\ f(t), & \text { if } \alpha=\beta, \\ D^{\beta-\alpha} f(t), & \text { if } \alpha<\beta,\end{cases}
$$

(v) ${ }^{C} D_{0^{+}}^{\beta} D^{m} f(t)=D^{\beta+m} f(t), m=0,1,2, \ldots, n-1<\beta<n$.

Definition 2.5 (see $[3,16]$ ). The Riemann-Liouville fractional integral operator of order $\rho \geq 0$ for a function $f \in C_{\mu},(\mu \geq-1)$ is defined as

$$
K^{\rho} f(x)=\frac{1}{\Gamma(\rho)} \int_{0}^{x}(x-t)^{\rho-1} f(t) d t, \quad \rho>0, x>0, \quad K^{0} f(x)=f(x)
$$

having the properties

$$
\begin{gathered}
K^{\rho} K^{\beta} f(x)=K^{\rho+\beta} f(x), \\
K^{\rho} x^{\beta}=\frac{\Gamma(\beta+1)}{\Gamma(\rho+\beta+1)} x^{\alpha+\beta} .
\end{gathered}
$$


According to the Caputo's derivatives, we obtain the following expressions:

$$
\begin{gathered}
{ }^{C} D^{\mu} C=0, \quad C=\text { constant }, \\
{ }^{C} D^{\mu} t^{\beta}= \begin{cases}\frac{\Gamma(\beta+1)}{\Gamma(\alpha+\beta+1)} t^{\beta-\alpha}, & \beta>\alpha-1, \\
0, & \beta \leq \alpha-1 .\end{cases}
\end{gathered}
$$

Lemma 2.6. If $m-1<\alpha \leq m, m \in N, f \in C_{\mu}^{m}, \mu \geq-1$, then the following two properties hold:

$$
\text { (1) } D^{\alpha} K^{\alpha} f(t)=f(t), \quad(2)\left(D^{\alpha} K^{\alpha}\right) f(t)=f(t)-\sum_{k=0}^{m-1} f^{(k)}\left(0^{+}\right) \frac{t^{k}}{k !} \text {. }
$$

In fact, Kilıçman and Zhour introduced the Kronecker convolution product and expanded to the Riemann-Liouville fractional integrals of matrices by using the Block Pulse operational matrix as follows:

$$
\frac{1}{\Gamma(\alpha)} \int_{0}^{t}\left(t-t_{1}\right)^{\alpha-1} \phi_{m}\left(t_{1}\right) d t_{1} \simeq F_{\alpha} \phi_{m}(t)
$$

where

$$
F_{\alpha}=\left(\frac{b}{m}\right)^{\alpha} \frac{1}{\Gamma(\alpha+2)}\left[\begin{array}{ccccc}
1 & \xi_{2} & \xi_{3} & \cdots & \xi_{m} \\
0 & 1 & \xi_{2} & \cdots & \xi_{m-1} \\
0 & 0 & 1 & \cdots & \xi_{m-2} \\
0 & 0 & 0 & \ddots & \vdots \\
0 & 0 & 0 & 0 & 1
\end{array}\right]
$$

see [23].

In our work, we consider Caputo fractional derivatives and apply the homotopy method in order to derive an approximate solutions of the fractional integrodifferential equations.

\section{Homotopy Method}

To illustrate the basic ideas of this method, we consider the following nonlinear differential equation:

$$
A(u)+f(\mathbf{r})=0, \quad \mathbf{r} \in \boldsymbol{\Omega},
$$


with boundary conditions

$$
B\left(u, \frac{\partial u}{\partial n}\right)=0, \quad \mathbf{r} \in \Gamma
$$

where $A$ is a general differential operator, $B$ is a boundary operator, $f(\mathbf{r})$ is a known analytical function, and $\Gamma$ is the boundary of the domain $\Omega$, see [24].

In general, the operator $A$ can be divided into two parts $L$ and $N$, where $L$ is linear, while $N$ is nonlinear. Equation (3.1), therefore, can be rewritten as follows:

$$
L(u)+N(u)-f(\mathbf{r})=0 .
$$

By using the homotopy technique that was proposed by Liao in $[7,8]$, we construct a homotopy of (3.1) $v(\mathbf{r}, p): \Omega \times[0,1] \rightarrow \mathcal{R}$ which satisfies

$$
\mathscr{H}(v, p)=(1-p)\left[L(v)-L\left(u_{0}\right)\right]+p[A(v)+f(\mathbf{r})]=0, \quad p \in[0,1], \mathbf{r} \in \boldsymbol{\Omega},
$$

or

$$
\mathscr{H}(v, p)=L(v)-L\left(u_{0}\right)+p L\left(u_{0}\right)+p[N(v)-f(\mathbf{r})]=0,
$$

where $p \in[0,1]$ is an embedding parameter and $u_{0}$ is an initial approximation which satisfies the boundary conditions. By using (3.4) and (3.5), we have

$$
\begin{aligned}
& \mathscr{H}(v, 0)=L(v)-L\left(u_{0}\right)=0, \\
& \mathscr{H}(v, 1)=A(v)-f(\mathbf{r})=0 .
\end{aligned}
$$

The changing in the process of $p$ from zero to unity is just that of $v(\mathbf{r}, p)$ from $u_{0}$ to $u(\mathbf{r})$. In a topology, this is also

known deformation, further $L(v)-L\left(u_{0}\right)$ and $A(v)-f(\mathbf{r})$ are homotopic.

Now, assume that the solution of (3.4) and (3.5) can be expressed as

$$
v=v+p v_{1}+p^{2} v_{2}+\cdots
$$

The approximate solution of (3.1), therefore, can be readily obtained:

$$
u=\lim _{p \rightarrow 1} v=v_{0}+v_{1}+v_{2}+\cdots
$$

The convergence of the series of (3.8) has been proved in the $[25,26]$. 


\section{The Variational Iteration Method}

To illustrate the basic concepts of the VIM, we consider the following differential equation:

$$
L u+N u=g(x)
$$

where $L$ is a linear operator, $N$ is a nonlinear operator, and $g(x)$ is an nonhomogenous term; for more details, see [19].

According to the VIM, one construct a correction functional as follows:

$$
u_{n+1}(x)=u_{n}(x)+\int_{0}^{x} \lambda\left[L u_{n}(s)+N \tilde{u}_{n}(s)-g(s)\right] d s
$$

where $\lambda$ is a general Lagrange multiplier, which can be identified optimally via the variational theory, and the subscript $n$ denotes the order of approximation, $\tilde{u}_{u}$ is considered variation $[6,27]$, that is, $\delta \tilde{u}_{u}=0$.

\section{Analysis of VIM}

To solve the fractional integrodifferential equation (1.1) by using the variational iteration method, with boundary conditions (1.2), one can construct the following correction functional:

$$
y_{k+1}(x)=y_{k}(x)+\int_{0}^{t} \mu \sum_{k=0}^{\infty} P_{k} D_{*}^{\alpha} y(s) d s-\mu \tilde{g}_{k}(x)-\lambda \int_{a}^{b} \mu H(x, s) \tilde{y}_{k}(s) d s,
$$

where $\mu$ is a general Lagrange multiplier and $\tilde{g}_{k}(x)$ and $\tilde{y}_{k}(x)$ are considered as restricted variations, that is, $\delta \tilde{g}_{k}(x)=0$ and $\delta \tilde{y}_{k}(x)=0$.

Making the above correction functional stationary, the following conditions can be obtained:

$$
\delta y_{k+1}(x)=\delta y_{k}(x)+\int_{0}^{t}\left[\sum_{k=0}^{\infty} P_{k} \mu(s) \delta D_{*}^{\alpha} y(s)-\delta \tilde{g}_{k}(x)-\lambda \int_{a}^{b} H(x, s) \mu(s) \delta \tilde{y}_{k}(s) d s\right],
$$

having the boundary conditions as follows:

$$
1-\left.\mu^{\prime}(s)\right|_{x=s}=0,\left.\quad \mu(s)\right|_{x=s}=1 .
$$

The Lagrange multipliers can be identified as follows:

$$
\mu(s)=\frac{1}{2}(s-x) .
$$


Substituting the value of $\mu$ from (5.4) into correction functional of (5.1) leads to the following iteration formulae:

$$
\begin{aligned}
y_{k+1}(x)= & y_{k}(x)+\frac{\mu}{2 \Gamma(\alpha-1)} \int_{0}^{x}(x-s)^{\alpha-2}(s-x) \\
& \times\left[\int_{0}^{t} \sum_{k=0}^{\infty} P_{k} D_{*}^{\alpha} y(s) d s-\widetilde{g}_{k}(x)-\lambda \int_{a}^{b} H(x, s) \tilde{y}_{k}(s)\right] d s, \\
y_{k+1}(x)= & y_{k}(x)-\frac{\mu(\alpha-1)}{2 \Gamma(\alpha)} \int_{0}^{x}(x-s)^{\alpha-1} \\
\times & {\left[\int_{0}^{t} \sum_{k=0}^{\infty} P_{k} D_{*}^{\alpha} y(s) d s-\widetilde{g}_{k}(x)-\lambda \int_{a}^{b} H(x, s) \tilde{y}_{k}(s)\right] d s, }
\end{aligned}
$$

by applying formulae (2.4), we get

$$
y_{k+1}(x)=y_{k}(x)-\frac{(\alpha-1) K^{\alpha}}{2 \Gamma(\alpha)}\left[\int_{0}^{t} \mu \sum_{k=0}^{\infty} P_{k} D_{*}^{\alpha} y(v) d v-\mu \tilde{g}_{k}(x)-\lambda \int_{a}^{b} \mu H(x, v) \tilde{y}_{k}(v)\right] d v
$$

The initial approximation can be chosen in the following manner which satisfies initial boundary conditions (1.2)-(1.3):

$$
y_{0}(x)=v_{0}+v_{1} x, \quad \text { where } v_{1}=D_{*}^{\alpha} y(0), v_{0}=D_{*}^{\alpha} y(a)
$$

We can obtain the following first-order approximation by substitution of (5.7) into

$$
y_{1}(x)=y_{0}(x)-\frac{(\alpha-1) K^{\alpha}}{2 \Gamma(\alpha)}\left[\int_{0}^{t} \mu \sum_{k=0}^{N} P_{k} D_{*}^{\alpha} y(v) d v-\mu \widetilde{g}_{0}(x)-\lambda \int_{a}^{b} \mu H(x, v) \tilde{y}_{k}(v)\right] d v .
$$

Substituting the constant value of $v_{0}$ and $v_{1}$ in the expression (5.8) results in the approximation solution of (1.1)-(1.3).

\section{Analysis of HPM}

This section illustrates the basic of HPM for fractional Fredholm integrodifferential equations with constant coefficients (1.1) with initial-boundary conditions (1.2).

In view of HPM $[25,26]$, construct the following homotopy for (1.1):

$$
\sum_{k=0}^{\infty} P_{k} D_{*}^{\alpha} y(x)=p\left[\sum_{k=0}^{\infty} P_{k} D_{*}^{\alpha} y(x)+\left(g(t)-\lambda \int_{a}^{b} H(x, t) y(x) d x\right)\right] .
$$



series in $p$ :

In view of basic assumption of HPM, solution of (1.1) can be expressed as a power

$$
y(x)=D_{*}^{\alpha} y_{0}(x)+p D_{*}^{\alpha} y_{1}(x)+p^{2} D_{*}^{\alpha} y_{2}(x)+p^{3} D_{*}^{\alpha} y_{3}(x)+\cdots
$$

If we put $p \rightarrow 1$ in (6.2), we get the approximate solution of (1.1):

$$
y(x)=D_{*}^{\alpha} y_{0}(x)+D_{*}^{\alpha} y_{1}(x)+D_{*}^{\alpha} y_{2}(x)+D_{*}^{\alpha} y_{3}(x)+\cdots .
$$

The convergence of series (6.3) has been proved in [28].

Now, we substitute (6.2) into (6.1); then equating the terms with identical power of $p$, we obtain the following series of linear equations:

$$
\begin{aligned}
& p^{0}: \sum_{k=0}^{\infty} P_{k} D_{*}^{\alpha} y_{0}(t)=0, \\
& p^{1}: \sum_{k=0}^{\infty} P_{k} D_{*}^{\alpha} y_{1}(t)=\sum_{k=0}^{\infty} P_{k} D_{*}^{\alpha} y_{0}(t)-\lambda \int_{a}^{b} H(x, t) y_{0}(x) d x, \\
& p^{2}: \sum_{k=0}^{\infty} P_{k} D_{*}^{\alpha} y_{2}(t)=\sum_{k=0}^{\infty} P_{k} D_{*}^{\alpha} y_{1}(t)+g(x)-\lambda \int_{a}^{b} H(x, t) y_{1}(x) d x, \\
& p^{3}: \sum_{k=0}^{\infty} P_{k} D_{*}^{\alpha} y_{3}(t)=\sum_{k=0}^{\infty} P_{k} D_{*}^{\alpha} y_{2}(t)-\lambda \int_{a}^{b} H(x, t) y_{2}(x) d x, \\
& p^{4}: \sum_{k=0}^{\infty} P_{k} D_{*}^{\alpha} y_{4}(t)=\sum_{k=0}^{\infty} P_{k} D_{*}^{\alpha} y_{3}(t)-\lambda \int_{a}^{b} H(x, t) y_{3}(x) d x,
\end{aligned}
$$

with the initial-boundary conditions

$$
D_{*}^{\alpha} y(a)=y(0), \quad D_{*}^{\alpha} y(0)=y^{\prime}(a)
$$

We can also take the initial approximation in the following manner which satisfies initial-boundary conditions (1.2)-(1.3):

$$
y_{0}(x)=v_{0}+v_{1} x, \quad \text { where } v_{1}=D_{*}^{\alpha} y(0), \quad v_{0}=D_{*}^{\alpha} y(a) .
$$

Note that (6.4) can be solved by applying the operator $K^{\beta}$, which is the inverse of operator $D^{\alpha}$ we approximate the series solution of HPM by the following $n$-term truncated series [29]:

$$
\chi_{n}(x)=D_{*}^{\alpha} y_{0}(x)+D_{*}^{\alpha} y_{1}(x)+D_{*}^{\alpha} y_{2}(x)+D_{*}^{\alpha} y_{3}(x)+\cdots+D_{*}^{\alpha} y_{n-1}(x),
$$

which results, the approximate solutions of (1.2)-(1.3). For further analysis, the variational iteration method, see [30] and the algorithm by the homotopy perturbation method, see [31]. 


\section{Conclusion}

The proposed methods are used to solve fractional Fredholm integrodifferential equations with constant coefficients. Comparison of the results obtained by the present method with that obtained by other method reveals that the present method is very effective and convenient. Unfortunately, the disadvantage of the second method is that the embedding parameter $p$ is quite casual, and often enough the approximations obtained by this method will not be uniform. So, in our future work we expect to study this kind of equation by using a combination of the variational iteration method and the homotopy perturbation method which has shown reliable results in supplying analytical approximation that converges very rapidly. However, we note that the papers [32, 33] suggest alternative ways for similar problems.

\section{Acknowledgments}

The first author would like to thank Professor Juan J. Trujillo for the very helpful discussion. The authors also acknowledge that this research was partially supported by University Putra Malaysia under the Research University Grant Scheme 05-01-09-0720RU.

\section{References}

[1] R. Hilfert, Applications of Fractional Calculus in Physics, World Scientific, Singapore, 2000.

[2] K. B. Oldham and J. Spanier, The Fractional Calculus, vol. 198 of Mathematics in Science and Engineering, Academic Press, 1974.

[3] I. Podlubny, Fractional Differential Equations, vol. 198 of Mathematics in Science and Engineering, Academic Press, San Diego, Calif, USA, 1999.

[4] A. Kadem and D. Baleanu, "Fractional radiative transfer equation within Chebyshev spectral approach," Computers \& Mathematics with Applications, vol. 59, no. 5, pp. 1865-1873, 2010.

[5] A. Kadem and D. Baleanu, "Analytical method based on Walsh function combined with orthogonal polynomial for fractional transport equation," Communications in Nonlinear Science and Numerical Simulation, vol. 15, no. 3, pp. 491-501, 2010.

[6] J. H. He, "Variational iteration method for delay differential equations," Communications in Nonlinear Science and Numerical Simulation, vol. 2, no. 4, pp. 235-236, 1997.

[7] S. J. Liao, "An approximate solution technique not depending on small parameters: a special example," International Journal of Non-Linear Mechanics, vol. 30, no. 3, pp. 371-380, 1995.

[8] S. J. Liao, "Boundary element method for general nonlinear differential operators," Engineering Analysis with Boundary Elements, vol. 20, no. 2, pp. 91-99, 1997.

[9] M. J. Ablowitz and P. A. Clarkson, Solitons, Nonlinear Evolution Equations and Inverse Scattering, vol. 149, Cambridge University Press, New York, NY, USA, 1991.

[10] G. Adomian, Nonlinear Stochastic Systems Theory and Applications to Physics, vol. 46, Kluwer Academic Publishers, Dordrecht, The Netherlands, 1989.

[11] Y. Chen and Z. Yan, "Weierstrass semi-rational expansion method and new doubly periodic solutions of the generalized Hirota-Satsuma coupled KdV system," Applied Mathematics and Computation, vol. 177, no. 1, pp. 85-91, 2006.

[12] E. Fan, "Extended tanh-function method and its applications to nonlinear equations," Physics Letters A, vol. 277, no. 4-5, pp. 212-218, 2000.

[13] Y. Luchko and R. Gorenflo, "An operational method for solving fractional differential equations with the Caputo derivatives," Acta Mathematica Vietnamica, vol. 24, no. 2, pp. 207-233, 1999.

[14] S. Momani and Z. Odibat, "Numerical approach to differential equations of fractional order," Journal of Computational and Applied Mathematics, vol. 207, no. 1, pp. 96-110, 2007.

[15] S. Kempfle and H. Beyer, "Global and causal solutions of fractional differential equations," in Proceedings of the 2nd International Workshop on Transform Methods and Special Functions, pp. 210-216, Science Culture Technology Publishing, Varna, Bulgaria, 1996. 
[16] S. G. Samko, A. A. Kilbas, and O. I. Marichev, Fractional Integrals and Derivatives: Theory and Applications, Gordon and Breach, Yverdon, Switzerland, 1993.

[17] Yu. F. Luchko and H. M. Srivastava, "The exact solution of certain differential equations of fractional order by using operational calculus," Computers $\mathcal{E}$ Mathematics with Applications, vol. 29, no. 8, pp. 73-85, 1995.

[18] B. J. West, M. Bologna, and P. Grigolini, Physics of Fractal Operators, Springer, New York, NY, USA, 2003.

[19] J. H. He, "Variational iteration method-a kind of non-linear analytical technique: some examples," International Journal of Non-Linear Mechanics, vol. 34, no. 4, pp. 699-708, 1999.

[20] J. H. He., S. K. Elagan, and Z. B. Li, "Geometrical explanation of the fractional complex transform and derivative chain rule for fractional calculus," Physics Letters A, vol. 376, no. 4, pp. 257-259, 2012.

[21] A. Le Méhauté, A. El Kaabouchi, and L. Nivanen, "Contribution of non integer Integro-differential operators(NIDO) to the geometrical undersanding of Riemann's conjecture-(I)," in Proceedings of the 2nd IFAC workshop on Fractional Differentiation and Its Applications, vol. 2, pp. 230-233, 2006.

[22] A. A. Kilbas, H. M. Srivastava, and J. J. Trujillo, Theory and Applications of Fractional Differential Equations, vol. 204 of North-Holland Mathematics Studies, Elsevier Science, Amsterdam, The Netherlands, 2006.

[23] A. Kilıçman and Z. A. A. Al Zhour, "Kronecker operational matrices for fractional calculus and some applications," Applied Mathematics and Computation, vol. 187, no. 1, pp. 250-265, 2007.

[24] J. H. He, "Homotopy perturbation method: a new nonlinear analytical technique," Applied Mathematics and Computation, vol. 135, no. 1, pp. 73-79, 2003.

[25] J. H. He, "Homotopy perturbation technique," Computer Methods in Applied Mechanics and Engineering, vol. 178, no. 3-4, pp. 257-262, 1999.

[26] J. H. He, "A coupling method of a homotopy technique and a perturbation technique for non-linear problems," International Journal of Non-Linear Mechanics, vol. 35, no. 1, pp. 37-43, 2000.

[27] J. H. He, "Some asymptotic methods for strongly nonlinear equations," International Journal of Modern Physics B, vol. 20, no. 10, pp. 1141-1199, 2006.

[28] J. H. He, Non-pertubative methods for strongly nonlinear problems, dissertation, GmbH, Berlin, Germany, 2006.

[29] Y. Nawaz, "Variational iteration method and homotopy perturbation method for fourth-order fractional integro-differential equations," Computers and Mathematics with Applications, vol. 61, no. 8, pp. 2330-2341, 2011.

[30] J. H. He, "A short remark on fractional variational iteration method," Physics Letters A, vol. 375, no. 38, pp. 3362-3364, 2011.

[31] H. E. Ji-Huan, "A Note on the homotopy perturbation method," Thermal Science, vol. 14, no. 2, pp. 565-568, 2010.

[32] A. Yildirim, S. A. Sezer, and Y. Kaplan, "Numerical solutions of fourth-order fractional integrodifferential equations," Zeitschrift fur Naturforschung A, vol. 65, no. 12, pp. 1027-1032, 2010.

[33] S. Yüzbasi, N. Sahin, and A. Yildirim, "A collocation approach for solving high-order linear Fredholm-Volterra integro-differential equations," Mathematical and Computer Modelling, vol. 55, no. 3-4, pp. 547-563, 2012. 


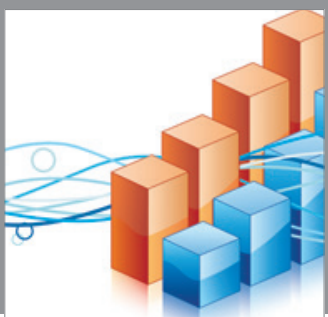

Advances in

Operations Research

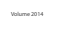

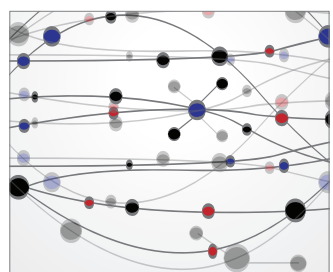

\section{The Scientific} World Journal
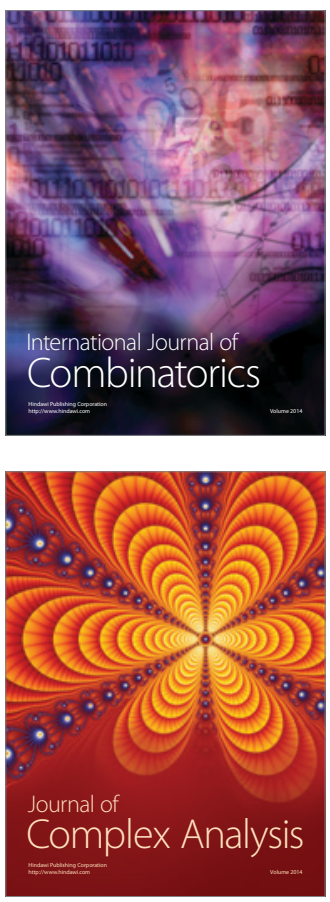

International Journal of

Mathematics and

Mathematical

Sciences
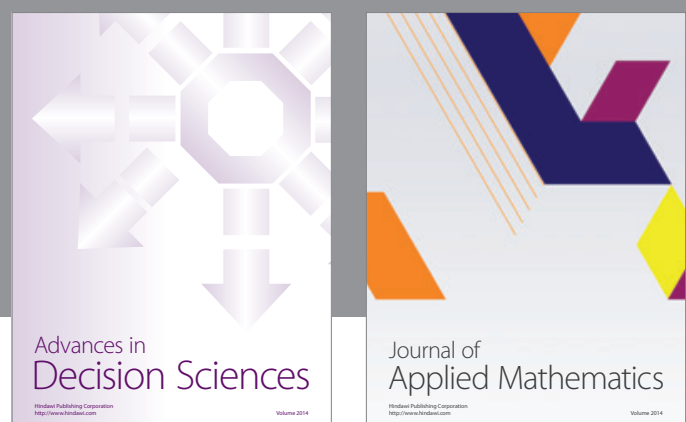

Journal of

Applied Mathematics
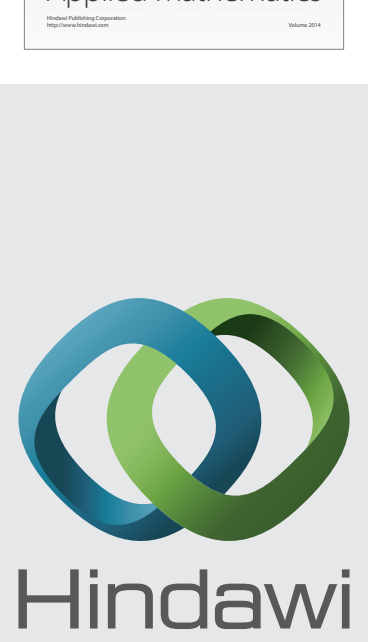

Submit your manuscripts at http://www.hindawi.com
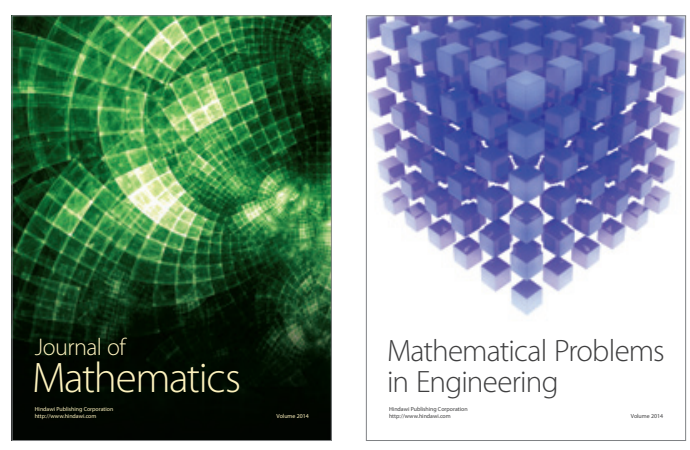

Mathematical Problems in Engineering
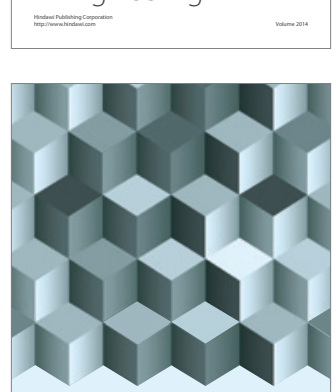

Journal of

Function Spaces
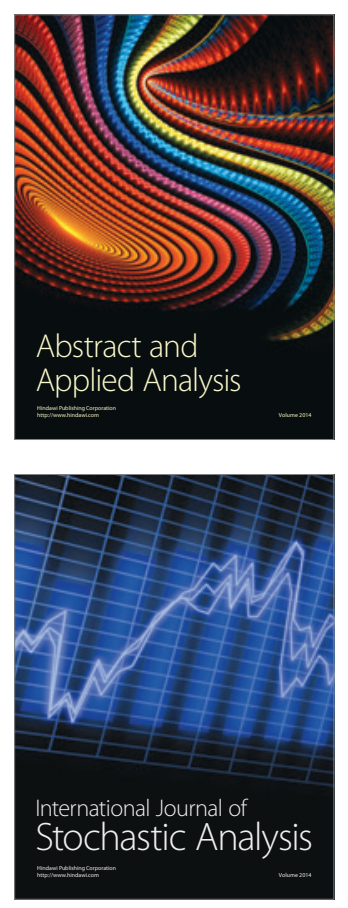

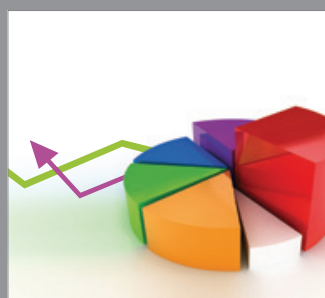

ournal of

Probability and Statistics

Promensencen
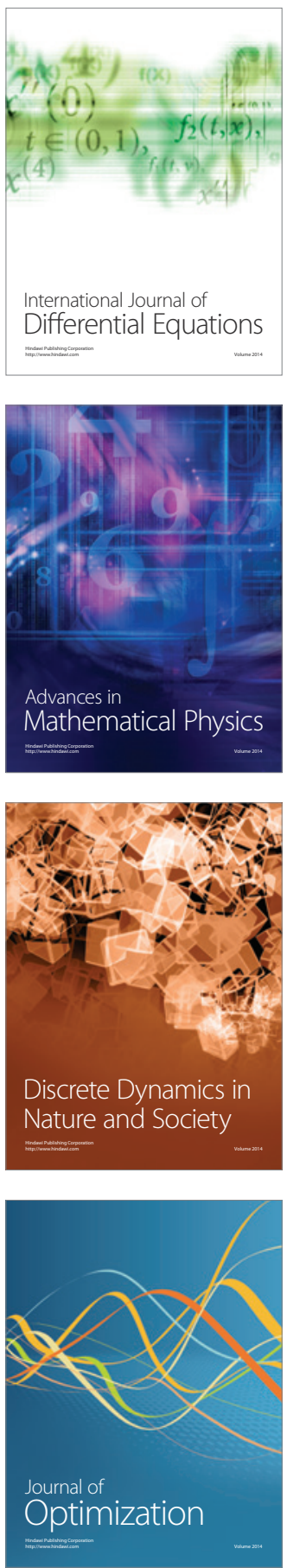